\title{
SambVca 2. A Web Tool for Analyzing Catalytic Pockets with Topographic Steric Maps
}

\author{
Laura Falivene, ${ }^{\dagger}$ Raffaele Credendino, ${ }^{\dagger}$ Albert Poater, ${ }^{\dagger,}$ Andrea Petta, ${ }^{\S}$ Luigi Serra, ${ }^{\S}$ Romina Oliva, $"$ \\ Vittorio Scarano, ${ }^{\S}$ and Luigi Cavallo*, ${ }^{\dagger}$
}

${ }^{\dagger}$ Physical Sciences \& Engineering Division (PSE), KAUST Catalysis Center (KCC), King Abdullah University of Science and Technology (KAUST), Thuwal, 23955-6900, Saudi Arabia

${ }^{\ddagger}$ Institut de Química Computacional i Catàlisi and Departament de Química, Universitat de Girona, Girona 17003, Spain

${ }^{\S}$ Dipartimento di Informatica ed Applicazioni, University of Salerno, Fisciano (SA), Italy

"Department of Sciences and Technologies, University "Parthenope" of Naples, Centro Direzionale Isola C4, Naples 80143, Italy

\section{Supporting Information}

ABSTRACT: Developing more efficient catalysts remains one of the primary targets of organometallic chemists. To accelerate reaching this goal, effective molecular descriptors and visualization tools can represent a remarkable aid. Here, we present a Web application for analyzing the catalytic pocket of metal complexes using topographic steric maps as a general and unbiased descriptor that is suitable for every class of catalysts. To show the broad applicability of our approach, we

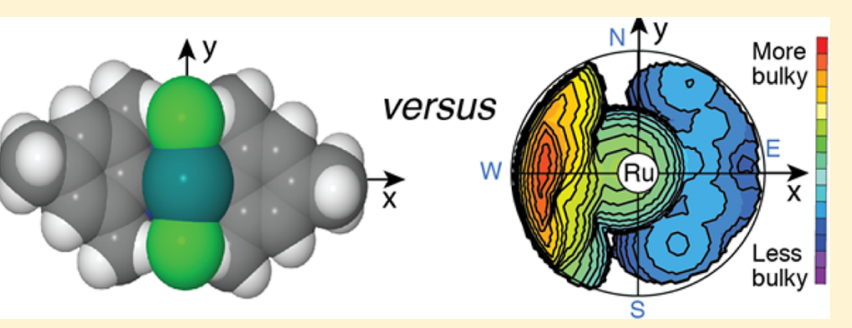
first compared the steric map of a series of transition metal complexes presenting popular mono-, di-, and tetracoordinated ligands and three classic zirconocenes. This comparative analysis highlighted similarities and differences between totally unrelated ligands. Then, we focused on a recently developed Fe(II) catalyst that is active in the asymmetric transfer hydrogenation of ketones and imines. Finally, we expand the scope of these tools to rationalize the inversion of enantioselectivity in enzymatic catalysis, achieved by point mutation of three amino acids of mononuclear $p$-hydroxymandelate synthase.

\section{INTRODUCTION}

Selecting the best catalyst in a disorganized catalyst space, composed of transition metal complexes, is a challenging task often driven by trial and error, or intuition, rather than a rational science. A classic solution to the problem is to use molecular descriptors capable of reorganizing the catalysts space as shown in Figure 1, paving the route to in silico design
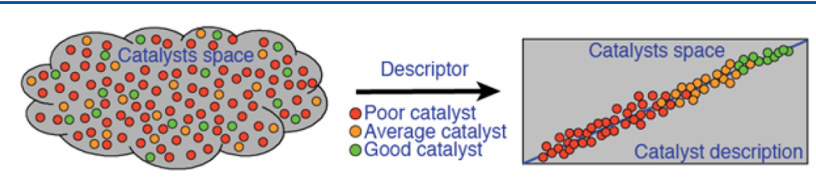

Figure 1. Representation of the potential of molecular descriptors in rationalizing catalyst behavior.

of new catalysts. ${ }^{1-5}$ The scope of these descriptors is to quantitatively correlate properties of the catalytic pocket (defined by analogy to enzymatic catalysis as the space around the active metal center) to how it behaves experimentally.

In a transition metal complex, the metal and the way the ligand wraps around the metal are fundamental to determining stability, activity, and selectivities (e.g., chemo, regio, and stereo) of the resulting catalyst. Therefore, developing a good catalyst means properly shaping the catalytic pocket with the caveat that good selectivity (i.e., greater than 95\%) typically means favoring one reaction pathway over the others by roughly $2 \mathrm{kcal} / \mathrm{mol}^{6}$ As a consequence, the challenge is to develop descriptors capable of capturing small structural differences in the catalytic pocket, which result in energy pathways differing by roughly $2 \mathrm{kcal} / \mathrm{mol}$.

Molecular descriptors are usually split into two categories depending on whether they influence catalytic behavior by altering the electronic status at the metal (i.e., electronic effects) or by constraining the space available to reactants (i.e., steric effects). ${ }^{7}$ Here, we focus on steric descriptors, of which one of the most successful is the Tolman cone angle, shown in Figure 2a. ${ }^{7}$ This popular descriptor appears in organometallic
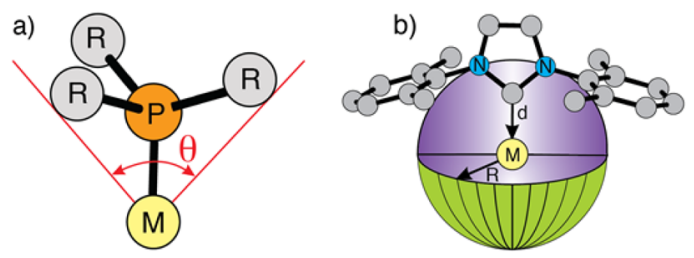

Figure 2. Representation of the Tolman cone angle (a) and the buried volume (b) steric descriptors.

Received: May 9, 2016

Published: June 27, 2016 
textbooks, although its application is limited to only phosphines. Examples of more general steric descriptors, not biased toward a specific class of ligands, are those proposed by Taft, ${ }^{8}$ Charton,, 10 and Verloop, ${ }^{11,12}$ as well as the buried volume descriptor we introduced to quantify the steric hindrance of $\mathrm{N}$-heterocyclic carbenes (NHCs); see Figure 2 b. ${ }^{13-16}$

Almost all the descriptors proposed to date condense the characteristics of a given catalyst into a single number, oversimplifying the complex chemical behavior that is often related to the specific shape of the catalytic pocket cast by a given ligand. In the case of asymmetric synthesis, this point becomes pivotal because the selectivity of the catalyst is related to the effective desymmetrization of the catalytic pocket. Singlevalue descriptors, such as the Tolman cone angle or the buried volume, are unable to capture these features. Thus, alternatives, such as the stereocartography methodology, ${ }^{17}$ the accessible molecular surface, ${ }^{18}$ and the successful set of sterimol parameters, have been proposed. ${ }^{5,19}$ Although powerful for quantifying differences in the catalytic pocket and for quantitatively correlating experimental behavior with the structure of a catalyst, these approaches do not provide a clear shape of the catalytic pocket. To fill this gap we introduced topographic steric maps; ${ }^{20}$ see Figure 3. These maps
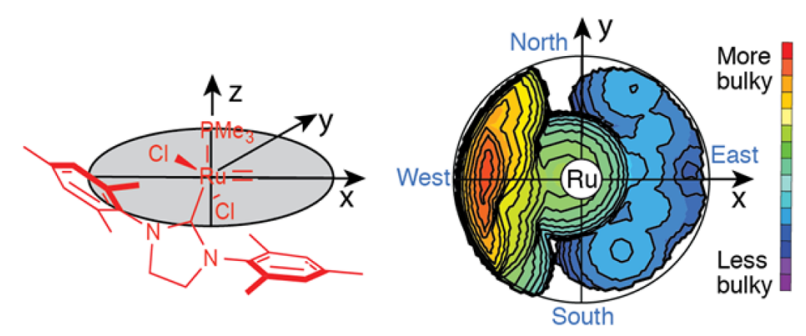

Figure 3. Steric map of a $(\mathrm{NCH})\left(\mathrm{Cl}_{2}\right) \mathrm{Ru}=\mathrm{CH}_{2}$ complex. The complex is oriented according to the scheme on the left; the steric map is reported on the right.

highlight the interaction surface between the catalyst and the substrate, with altimetry isocontour lines offering a quantitative description of the catalytic pocket. Similar to geographical maps, topographic steric maps can be focused on hemispheres (northern/southern or eastern/western) or quadrants (e.g., northwestern (NW) or southeastern (SE)).

The successful use of topographic steric maps to describe NHC-based ligands and the reactivity they promote ${ }^{21-23}$ encouraged us to test the application of these maps to nonNHC-based ligands. We were able to justify the inversion of regioselectivity in the Mizoroki-Heck reaction promoted by Pd-catalysts, ${ }^{24}$ the stereoselectivity in propene oxide polymerization promoted by bimetallic Co-salen complexes, ${ }^{25}$ the cis/ trans selectivity in olefin epoxidation promoted by $\mathrm{Ru}-$ catalysts, ${ }^{26}$ and the stereoselectivity in group 4 propene polymerization catalysts. ${ }^{27}$ Finally, we have even used this kind of map in organocatalyzed reactions, such as stereoselective Michael addition. $^{28}$

As our confidence in the applicability of topographic steric maps expanded to a variety of different systems, we decided to develop a free Web tool for generating topographic steric maps. The new Web tool, SambVca 2, replaces the SambVca $1 \mathrm{Web}$ tool, which was developed to calculate the buried volume descriptor. This newer version can calculate the buried volume while simultaneously generating a topographic steric map. It has a graphical interface designed to improve interaction between the Web tool and the user. To show the applicability of steric maps as a versatile tool able to provide a fingerprint of basically any ligand used in homogeneous catalysis, we apply it to a series of typical ligands used in organometallic chemistry. In the spirit of the Tolman and sterimol approaches, the main philosophy under the development of topographic steric maps is their intuitiveness, provided that a crystallographic or computed structure is available.

We have divided the considered examples into two main sections. In the first, we use topographic steric maps to characterize the catalytic pocket of prototype complexes containing a variety of very popular ligands. The chemistry and the reaction mechanism behind these ligands have been characterized in several studies. ${ }^{29-36}$ Here, we contribute a straightforward and consistent comparison of the catalytic pocket in these totally unrelated catalysts, evidencing that steric maps can be applied to basically any complex. In the second section, we apply topographic steric maps to analyze the catalytic pocket in two systems active in asymmetric catalysis. Finally, we show that steric maps can also be applied to a metalloenzyme, which would extend the scope of steric maps to the analysis of metalloproteins.

\section{METHODS}

Geometry Optimization of Systems 1-21. All density functional theory calculations were performed at the GGA level with the Gaussian09 program ${ }^{37}$ using the BP86 functional of Becke and Perdew. ${ }^{38}$ The electronic configuration of the molecular systems was described with the standard triple- $\zeta$ basis set with a polarization function proposed by Ahlrichs and co-workers for main group atoms (TZVP keyword in Gaussian09). ${ }^{39}$ For transition metals, we used the small-core, quasi-relativistic Stuttgart/Dresden effective core potential with the associated triple- $\zeta$ valence basis set (standard SDD keywords in Gaussian09). ${ }^{40}$ All complexes were considered in the singlet electronic state with the exception of the Mn-based systems, for which a triplet state was considered. ${ }^{41-45}$

Calculation of Steric Maps. To build the steric map, the overall complex was oriented in a Cartesian frame with a selected point at the origin, a second point along the $z$-axis, and a third point in the $x z$ plane. These three points are understood as corresponding to an atom or to the midpoint of a chosen subset of atoms. For example, the complex in Figure 4 was oriented with the metal center at the origin,

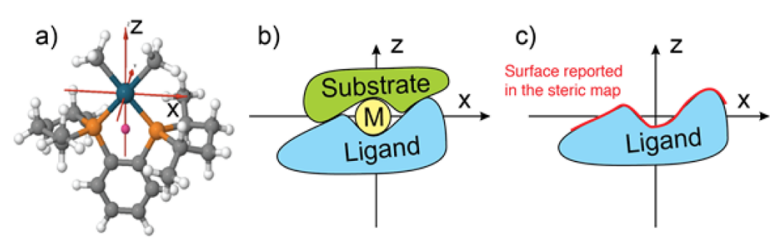

Figure 4. Example of the complex orientation (a) of the interaction between the ligand and the substrate (b) and of the surface reported in steric maps (c).

the midpoint of the two $\mathrm{P}$ atoms along the negative direction of the $z$ axis, and one of the $\mathrm{P}$ atoms in the $x z$-plane; see Figure 4a. After alignment of the complex, atoms that must be excluded from the steric map calculations were removed, and the first coordination sphere around the metal was analyzed. For example, the $\mathrm{PdMe}_{2}$ moiety was removed from the complex in Figure $4 \mathrm{a}$, and the MeDuPhos ligand was analyzed.

After the complex was oriented and the selected atoms were removed, a sphere of radius $R$, centered at the origin, is sectioned by a regular three-dimensional cubic mesh of spacing $s$, which defines cubic voxels $v$. The distance between the center of each voxel with all the 
atoms in the ligand was tested to check if any of the atoms were within the van der Waals distance from the center of the examined voxel. If no atom was within the van der Waals distance, the examined voxel was marked as a free voxel, and otherwise the examined voxel was marked as buried.

After all the voxels in the sphere are marked as free or buried for each $(x, y)$ point within the first coordination sphere, the program scans the sphere from the top (i.e., from positive $z$-values) to determine the $z$-value of the first buried voxel. This procedure results in a surface defined as $S(x, y)=z_{\mathrm{B}}$, which represents the surface of the ligand that is exposed toward the incoming reactants. In other words, this $S(x, y)=z_{\mathrm{B}}$ surface defines the shape of the reactive pocket. Positive values of $z_{\mathrm{B}}$ indicate that the ligands protrude in the $z>0$ halfsphere, which is the half-sphere where the reacting groups are placed. A schematic representation of the interaction surface between the catalyst and the substrate is shown in Figure $4 \mathrm{~b}$.

Finally, the maps are a simple two-dimensional isocontour representation of the interaction surface $S(x, y)=z_{\mathrm{B}}$, as shown in Figure $4 \mathrm{c}$. In this work, the radius $R$ of the sphere around the metal center was set to $3.5 \AA$, and a mesh of $0.1 \AA$ was used to scan the sphere for buried voxels. For the atoms, we adopted the van der Waals radii taken from the Cambridge Structural Database (CSD), which were taken from ref 46 . Although we did not consider hydrogen atoms to build the steric maps discussed here, for consistency with the CSD the hydrogen radius taken from ref 47 is included in the Web server database. Also in line with the CSD protocol, radii that were not available from either of these works were set to a default $2.00 \AA$ and scaled by $1.17 .^{13,15}$

The steric maps presented in this work were obtained using the SambVca 2 Web application. ${ }^{48}$ Since the graphical interface is limited to handling a maximum of 1000 atoms, the input file for generating the catalytic pocket of Amycalotopsis orientalis and of the S221M/V223F/ Y359A mutant of Streptomyces coelicolor, Protein Data Bank entries

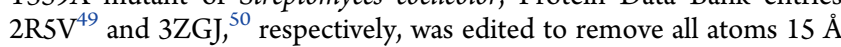
away from the metal center. The steric maps were then generated with SambVca 2 after uploading these manipulated files.

\section{RESULTS}

Characterization and Comparison of Catalysts. In this section, we illustrate the variety in shape of catalytic pockets from different families of catalysts. We discuss a few cases of popular mono-, di-, and tetracoordinated ligands, and we complete this section by analyzing typical group 4 metallocenes. Ball-and-stick and space-filling (CPK) models of systems 1-18 are reported in Figure S1.

We start with the analysis of three monocoordinated ligands, comprising two of the most representative examples of phosphine and NHC systems (see Figure 5). Percent buried volume $\left(\% V_{\text {Bur }}\right)$ highlights a difference in steric bulkiness between these three ligands, with the $\mathrm{PCy}_{3}$ ligand in $\mathbf{1}$ clearly less bulky than the NHC ligand in $\mathbf{2}$ and 3. Interestingly, the steric maps illustrate that the same ligand can shape different catalytic pockets (see maps of $\mathbf{2}$ and $\mathbf{3}$ ). In the steric map of $\mathbf{2}$, the three $\mathrm{CO}$ moieties force the two aryl $\mathrm{N}$-substituents perpendicular to the NHC ring, producing a substantially flat steric map with small bulges at the periphery. Instead, the steric map of $\mathbf{3}$ is characterized by a SE to NW groove hosting the $\mathrm{CO}$ and $\mathrm{Cl}$ moieties cis to the NHC ligand; see Figure S1. This shape of the steric map is a consequence of a small rotation of the aryl N-substituents of the NHC ligand in 3. The difference in the steric map of the same NHC in $\mathbf{2}$ and $\mathbf{3}$ is in line with the remarkable flexibility of NHCs. ${ }^{21}$

Next, we analyze the steric map shaped by dicoordinated ligands in complexes 4-12; see Figure 6. In all cases, the middle point of the two coordinating atoms is oriented at negative values along the $z$-axis, as shown by Figure 4 a.

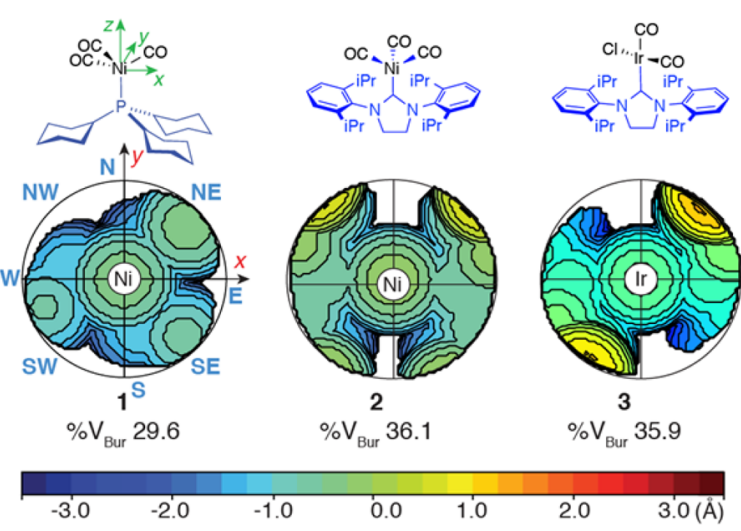

Figure 5. Steric maps of transition metal complexes with a monocoordinated ligand. The orientation of the ligand for $\mathbf{1}$ is consistent with the orientation of Figure $4 \mathrm{a}$, and it applies to all systems in this work. The labeling of quadrants as NE (northeast), SE (southeast), SW (southwest), and NW (northwest) is consistent with the labeling of Figure 3, and it applies to all the steric maps in this work.

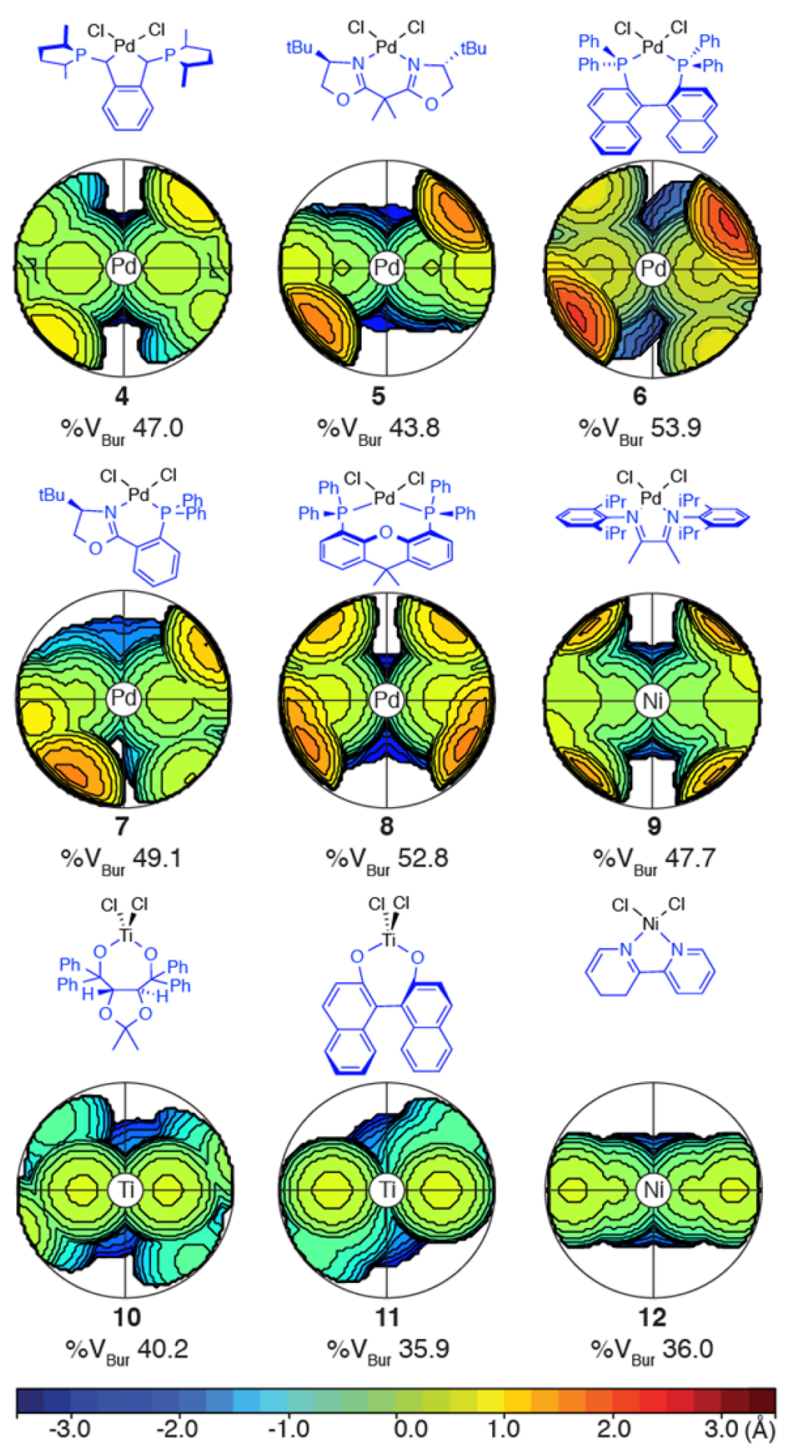

Figure 6. Steric map of transition metal complexes presenting a popular dicoordinated ligand. 
Complexes 4-7 contain privileged chiral ligands capable of conferring enantioselectivity over a variety of asymmetric reactions. ${ }^{34}$ The $C_{2}$-symmetric steric map of the MeDuPhos ligand $^{29}$ in 4 , with a $\% V_{\text {Bur }}$ of 47.0 , presents a flat catalytic pocket that covers nearly the entire area. The upward-oriented methyl groups correspond to the soft bulges in the SW and NE quadrants. Alternatively, the catalytic pocket of $\mathbf{5}$ shows the slim Box skeleton occupying space along the equator, with a \% $V_{\text {Bur }}$ of 43.8 , and tert-butyl groups that protrude out of the catalytic pocket in the SW and NW quadrants more than the methyl groups of 4 . The steric map of 6 shows a flat floor similar to 4 and a better defined SE to NW groove, shaped by the upward-pointing $\mathrm{Ph}$ groups. Interestingly, the catalytic pocket shaped by the nonsymmetric PHOX ligand of 7 also presents steric bulges in opposite quadrants, mimicking the catalytic pocket of 4-6. This variability in the catalytic pockets is not easily appreciated by analysis of the ball-and-stick or CPK models; see Figure S1.

Next, we examined the catalytic pocket designed by the Xantphos ligand in 8. The peculiar behavior of this family of catalysts has been ascribed to the large bite angle characterizing this ligand. ${ }^{36,51}$ The large bridge of $\mathbf{8}$ forces the ligand to assume a folded structure with the two phenyl substituents pointing up and facing each other in the southern hemisphere. The steric map of the diimine ligand in 9 shows how the four isopropyl ortho substituents block access to the axial coordination positions, leaving an east to west groove in the square planar coordination plane. ${ }^{52}$ Interestingly, the shape of the catalytic pocket in $\mathbf{9}$ is similar to that in $\mathbf{2}$.

The ligands in 10-12 have less impact in the proximity of the metal center. Complexes $\mathbf{1 0}$ and $\mathbf{1 1}$ have the TADDOL and the Binol ligands, respectively, and are used in Ti-catalyzed enantioselective synthesis. ${ }^{31}$ Within the accepted mechanism, the $\mathrm{Ti}$ center moves from tetrahedral to octahedral geometry during the course of the reaction, which implies that the TADDOL and Binol ligands have to leave space to host additional ligands. This explains the reduced space occupation of these ligands, with minor bulges protruding out of the plane containing the metal in the NW and SE quadrants. The last dicoordinating ligand we considered is the bipy ligand of 12 , a slim ligand occupying space only in the equatorial belt. This leaves space for the coordination of additional ligands, such as in the octahedral $\mathrm{Ru}^{2+}$ complexes active in photocatalytic water oxidation. ${ }^{53,54}$

Next, we compared the tetracoordinated O,N,N,O ligands of 13-15, see Figure 7. The first two systems have been found active in olefin epoxidation ${ }^{35}$ and the latter in stereoselective 1olefin polymerization. ${ }^{55}$ Comparison of 13 and 14 evidences the key role of the chiral diamino-cyclohexane bridge in $\mathbf{1 4}$ versus the diamino-aromatic bridge in 13. Specifically, the flat bridge of 13 results in a flat geometry of the whole salen ligand, with minor differences between the western and eastern hemispheres. The bulky tert-butyl groups prevent attack by the olefin on the $\mathrm{Mn}=\mathrm{O}$ bond from the south; the only channel available to approach the $\mathrm{Mn}=\mathrm{O}$ bond is from the north, which is above the aromatic bridge. Differently, the chiral bridge in $\mathbf{1 4}$ imparts a folding to the salen moiety, with the flap in the western hemisphere bent toward the $\mathrm{Mn}=\mathrm{O}$ moiety, preventing attack by the olefin from the west. ${ }^{35}$ At the same time, the salen flap in the eastern hemisphere is bent away from the $\mathrm{Mn}=\mathrm{O}$ moiety, opening the door for attack by the olefin from the east. The tert-butyl groups and the $\mathrm{N}, \mathrm{N}$-diaminocyclohexane groups prevent attack from the south and north,

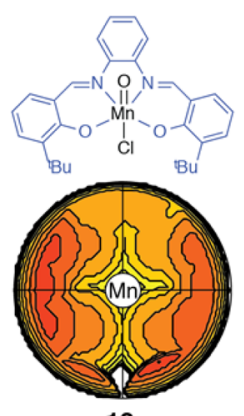

13

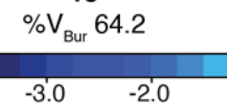

Figure 7. Steric map of transition metal complexes presenting a popular tetracoordinated ligand.

respectively. ${ }^{35}$ Comparison between 14 and 15 shows how similar ligands can shape different catalytic pockets by folding differently around the metal center: square planar in 14 versus $f a c-f a c$ in 15. As a result, the steric map of $\mathbf{1 5}$ shows a large groove in the equatorial belt to host the growing polymer chain and the monomer during isoselective polymerization of propene. $^{55-57}$

Finally, we focus on the three zirconocenes of Figure 8, also active in stereoselective propene polymerization. The steric

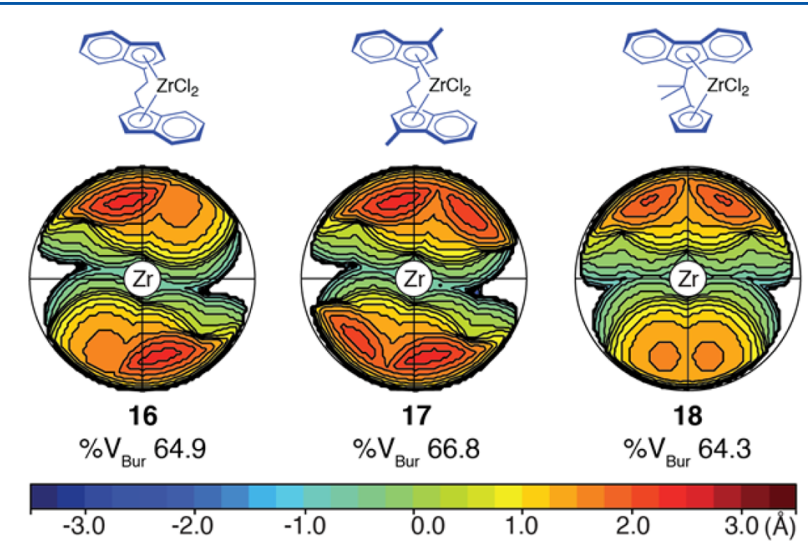

Figure 8. Steric map of typical zirconocenes.

map of the isoselective $C_{2}$-symmetric complex 16 presents the expected space occupation of less hindered NE and SW quadrants than NW and SE quadrants. Within the accepted model for stereoselective olefin polymerization, at each step the growing chain is oriented in one of the open quadrants (see Figure S2). ${ }^{57}$

The relationship between the tacticity of the resulting polymer and the symmetry of the metallocene, known as Ewen's symmetry rules, ${ }^{57,58}$ is well captured by comparing the steric map of 16 with those of 17 and 18. Focusing on 17, the $3,3^{\prime}$ methyl substituents of the bisindenyl skeleton induce formation of substantially atactic polypropylene, and the steric map of $\mathbf{1 7}$ shows similar hindrance in all four quadrants, preventing a chiral orientation of the growing chain. ${ }^{59}$ This comparison is completed by the steric map of the $C_{s}$-symmetric complex 18, which leads to syndiotactic polypropylene. As expected, the steric map of $\mathbf{1 8}$ shows hindrance only in the northern quadrants, resulting in enantiomeric transition states 
for propene insertion within a regular chain-migratory polymerization mechanism. ${ }^{60}$

Now that we have presented the potential of steric maps to illustrate known systems with well-established reaction pathways, we will now apply them to catalysts with less consolidated reaction mechanisms. It remains clear that a thorough understanding of these systems requires a detailed characterization of electronic structure methods of the complete reaction pathway.

Asymmetric Catalysis. Using steric maps, we investigate the source of enantioselectivity in asymmetric catalysis. To start, we compare recently developed amine(imine)diphosphine $\mathrm{Fe}(\mathrm{II})$ catalysts used for the asymmetric transfer hydrogenation of ketones and imines ${ }^{61}$ (see Figure 9a) to the

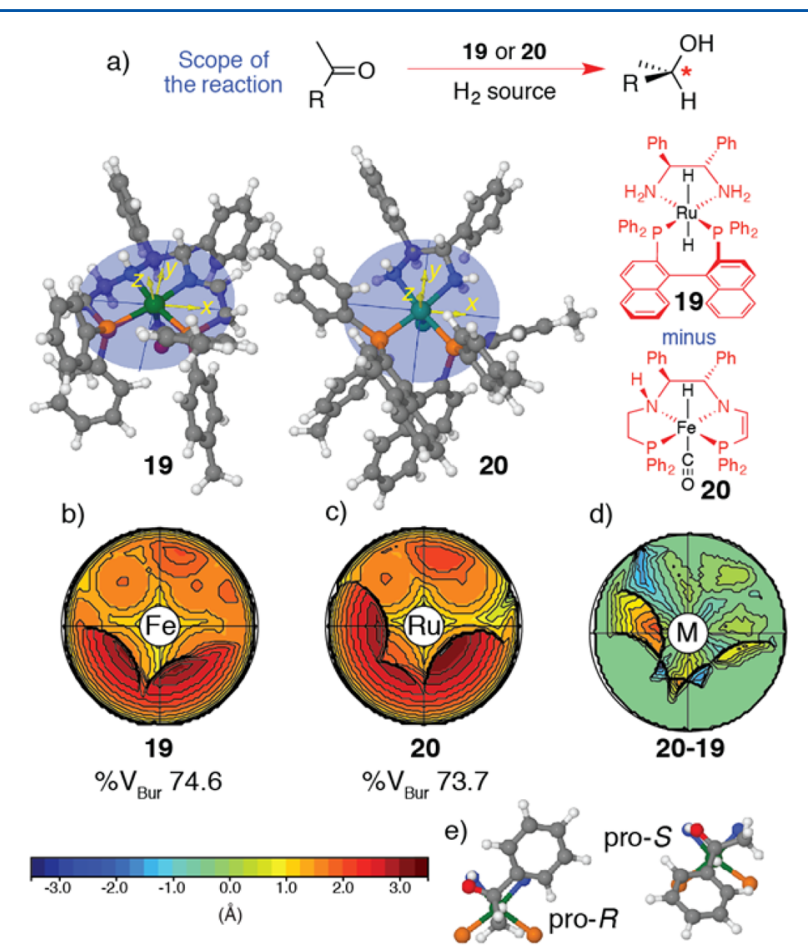

Figure 9. Scope of the reaction (a); ball-and-stick representations and steric maps of complexes 19 and 20 (b and c); line drawing representation of complexes 19 and 20 and difference steric map $20-$ 19 (d); pro- $R$ and pro- $S$ faces of acetophenone as interacting with the catalyst in the H-transfer transition state (e). For details on steric map calculations, see the legend in Figure 2. The geometries of the transition states of $(\mathrm{e})$ were derived from ref 66.

classic Noyori (binaphthol)(diamines) $\mathrm{Ru}(\mathrm{II})$ catalyst. ${ }^{62}$ Then, we expand the scope to metalloproteins by analyzing the catalytic pocket of a mononuclear $p$-hydroxymandelate synthase taken from $A$. orientalis, which naturally converts the substrate $p$-hydroxyphenylpyruvate into $S$-hydroxymandelate (see Figure 10 a). ${ }^{50,63,64}$

Amine(imine)diphosphine $\mathrm{Fe}(\mathrm{II})$ catalysts, such as 19 of Figure $9 \mathrm{~b}$, promote the synthesis of chiral alcohols and amines with enantiomeric excesses above $90 \%$ and turnover frequencies as high as $200 \mathrm{~s}^{-1}$ at room temperature. ${ }^{61,65}$ The enantioselective step is considered the transfer of a hydride from the Fe center to the substrate, a step assisted by a H-bond between the $\mathrm{N}-\mathrm{H}$ moiety of the ligand and the $\mathrm{C}=\mathrm{O}$ moiety of the substrate. In this framework, the steric map of Figure $9 \mathrm{~b}$ clearly shows the asymmetry of the catalytic pocket: the southern hemisphere is practically blocked by the bulky $\mathrm{PPh}_{2}$ a)

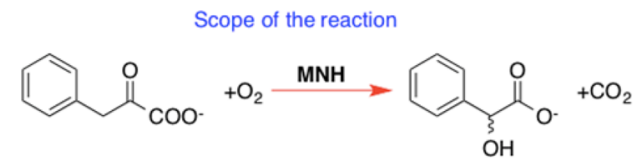

b) $\stackrel{\text { His }}{261}_{\vdots}$

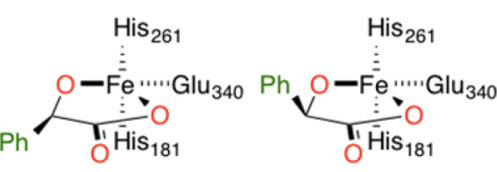

Active site of $\mathrm{MNH}$
S-product (WT product)

$R$-product (mutant product)

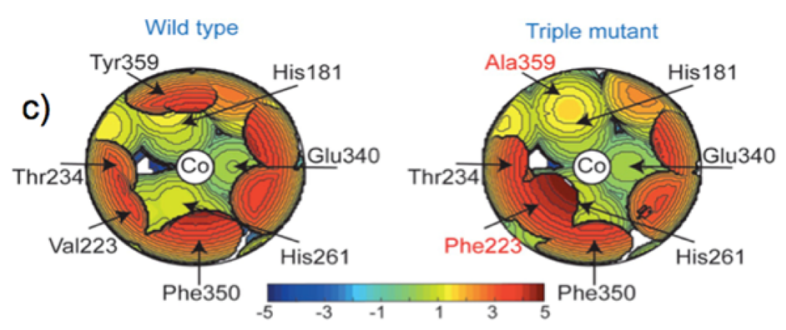

Figure 10. Scope of the reaction (a); schematic representation of the active site (b); and steric map of the active site in the crystallographic structure of the wild-type A. orientalis and of the S221M/V223F/ Y359A mutant of S. coelicolor (c).

groups. In the northern hemisphere, the chiral $(S, S)$-bridge forces the $\mathrm{N}-\mathrm{H}$ moiety in the NW quadrant to protrude from the coordination plane of the metal toward the substrate. This analysis of the catalytic pocket explains that during the transfer hydrogenation transition state the acetophenone pro- $R$ face of Figure 9e will be favored because it places the bulkier phenyl substituent in the less hindered NW quadrant of the steric map of Figure $9 \mathrm{~b}$. Hydrogenation of the acetophenone pro- $S$ face of Figure 9e is instead disfavored because the bulkier phenyl group clashes against the bulge provided by the $\mathrm{PPh}_{2}$ groups in the SW quadrant of the steric map of Figure $9 \mathrm{~b}$.

The steric map of the chiral pocket of the $(S, S)$-diamine $S$ BINAP catalyst 20 (see Figure 9c) shows a clear similarity with the steric map of 19: the $R$ product is preferentially formed in both 19 and 20. The similarity between the two catalytic pockets is further enhanced by analysis of the difference steric map of Figure 9d, which reports for each point of the map the difference between the steric map of 20 and that of 19. The difference map clearly shows that the only meaningful difference in the catalytic pocket of $\mathbf{1 9}$ and 20 is a slightly higher hindrance just above the equator in the NW quadrant of the steric map of 20.

Next, we compare the catalytic pockets of two enzymes with complementary enantioselectivity that belong to mononuclear nonheme $\mathrm{Fe}$ (II)-dependent hydroxylases. One is the wild-type mononuclear $p$-hydroxymandelate synthase (HMS) from $A$. orientalis, which catalyzes the selective formation of $(S)$ mandelate from phenylpiruvate (Figure 10a), and the other corresponds to the triple mutant (S221M/V223F/Y359A) of the homologous HMS from $S$. coelicolor, in silico designed to exhibit an inverted enantioselectivity (i.e., to favor the formation of the $(R)$-mandelate, starting from the same substrate). ${ }^{50,63,64}$

High-resolution crystal structures, including the enantiomeric reaction products coordinated to the metal center, are available for both of these enzymes and correspond to the PDB entries $2 \mathrm{R}^{2} \mathrm{~V}^{49}$ and $3 \mathrm{ZGJ},{ }^{50}$ respectively. In both structures, Co(II) 
was used as a redox-inactive $\mathrm{Fe}(\mathrm{II})$ mimic. The active site of these proteins presents nonideal trigonal bipyramidal geometry at the metal center with the $\mathrm{N}$ atoms of His 181 and His 261 in the equatorial plane and the $\mathrm{O}$ atom of Glu340 along the main axis (referring to the numbering of $S$. coelicolor residues, also used in the following). The other two coordination positions are available for catalysis and are occupied by the products $(S)$ mandelate and $(R)$-mandelate in the X-ray structures of the wild type and of the mutant, respectively. Upon orienting the metal center as in Figure $10 \mathrm{~b}$, the $\mathrm{Ph}$ group of $(R)$-mandelate will point up from their respective catalytic pockets.

Figure 10c shows the steric maps that characterize the catalytic pockets of these enzymes, when the metal center is oriented as in Figure 10b. Analysis of the maps evidences that the coordinating residues, His181, His261, and Glu340, are located, in both cases, at the bottom of the catalytic pocket. Space above the metal is instead constrained by several residues at positions 223, 234, 261, 340, 350, and 359 (in the wild type). The aromatic ring of $(S)$-mandelate is placed in the SW quadrant in the wild type, in an open subpocket between the metal and residues Thr234 and Val223, while that of $(R)$ mandelate is placed in the NW quadrant of the mutant catalytic pocket. (R)-Mandelate cannot be accommodated in the catalytic pocket of the wild-type because it would clash against the large Tyr359 residue. These steric maps clearly illustrate the impact that the Val223Phe and Tyr359Ala mutations have on determining the inverted enzyme enantioselectivity of the mutant. Indeed, the Val1223Phe mutation blocks the open space in the SW quadrant where the aromatic ring of $(S)$ mandelate would be placed, while the Tyr259Ala mutation opens space in the NW quadrant to accommodate the aromatic ring of $(R)$-mandelate.

Which Is the Right Size of the First Coordination Sphere? All analyses described above were made assuming a $3.5 \AA$ radius of the sphere around the metal center. With the exception of Figure 10, percent buried volume was calculated and the steric maps were generated using this radius. Figure 10 was an exception because bulkiness is not given by groups directly bound to the metal. The value of $3.5 \AA$ was selected as the optimal value to correlate the DFT dimerization energy of 33 N-heterocyclic carbenes in the Wanzlick equilibrium. ${ }^{15}$ Further, it proved to be a robust value capable of rationalizing a large amount of experimental data. Considering that typical metal-ligand bond distances are within $2.0-2.5 \AA$, a value of $3.5 \AA$ to define the first coordination sphere around the metal is expected to account for the space roughly comprising the van der Waals volume occupied by the atoms coordinated to the metal. While this is the default choice, we are aware that a different value may be more suitable for other studies, for example, in bimetallic systems, where reactivity occurs between substrates attached to two different metal atoms. ${ }^{25,26}$ The impact of the radius on the percent buried volume and the steric map is shown in Figure 11 using the PNN Ir-pincer complex 21 of Figure 11a, which effectively converts alcohols into esters and synthesizes amides from alcohols and amines. $^{67,68}$

Figure $11 \mathrm{~b}$ and $\mathrm{c}$ show minor differences between the percent buried volume and the steric map of $\mathbf{2 1}$ when spheres with radii of 3.5 and $4.0 \AA$ are compared; differences become more pronounced at a radius of $5.0 \AA$, as shown by Figure $11 \mathrm{~d}$. In particular, the difference in steric hindrance given by the ethyl groups on the $\mathrm{N}$ atom compared to the tert-butyl groups on the $\mathrm{P}$ atom becomes more evident. A similar conclusion is

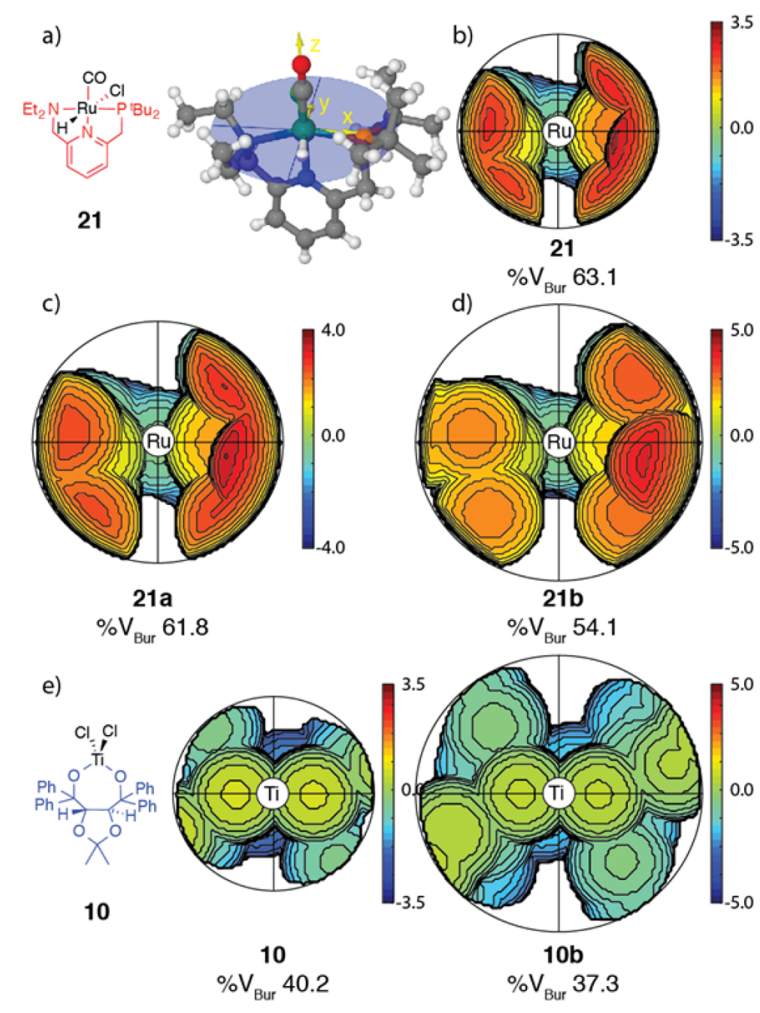

Figure 11. Structure of the Ir-pincer complex 21 (a); steric maps calculated with a sphere radius of 3.5, 4.0, and 5.0 Å, respectively (bd). Schematic representation of the Ti-TADDOL complex 10 and of the steric maps calculated with a sphere radius of 3.5 and $5.0 \AA$, respectively, (e).

reached when the catalytic pocket of the TADDOL system 10 is compared at radii of 3.5 and $5.0 \AA$, as shown by Figure 11e. Nevertheless, this technique has proven to successfully characterize the overall asymmetry and shape of the catalytic pocket independent from the radius. In this scenario, when bulkiness is not given by groups directly bound to the metal, as in the case discussed in Figure 10, a larger radius is necessary to thoroughly capture the shape of the catalytic pocket.

\section{CONCLUSIONS}

Classifying the behavior of catalysts using molecular descriptors is an exercise that can be useful for developing more effective catalysts. Here, we have presented the freely available Web application SambVca 2, which can be used to generate the topographic steric map characterizing the catalytic pocket around a metal center with any type of transition metal complex. SambVca 2 has a user-friendly graphical interface that also calculates the buried volume steric descriptor and is expected to completely replace SambVca 1 . The buried volume and the topographic steric maps are descriptors that are intended to be suitable for application to any system. This allows building an atlas including all classes of ligands. In addition, we showed that the scope of these tools could be expanded to investigate catalytic pockets of proteins, opening the door to a systematic analysis and classification of catalytic pockets of metalloenzymes. Finally, having defined the surface of catalytic pockets, it allows a systematic characterization of their properties: electrostatic potential, polar versus nonpolar regions, and patches for optimal dispersion interaction with the 
reacting groups. This information can then be used to optimize the complementarity between catalyst and substrate.

\section{ASSOCIATED CONTENT}

\section{S Supporting Information}

The Supporting Information is available free of charge on the ACS Publications website at DOI: 10.1021/acs.organomet.6b00371.

Ball-and-stick and CPK representation of complexes 118 (PDF)

Cartesian coordinates of complexes 1-18 (XYZ)

\section{AUTHOR INFORMATION}

\section{Corresponding Author}

*E-mail: luigi.cavallo@kaust.edu.sa.

\section{Notes}

The authors declare no competing financial interest.

\section{ACKNOWLEDGMENTS}

This work was supported by the King Abdullah University of Science and Technology (KAUST). A.P. thanks the Spanish MINECO for project CTQ2014-59832-JIN and European Commission for a Career Integration Grant (CIG09-GA-2011293900).

\section{REFERENCES}

(1) Fey, N. Dalton Trans. 2010, 39, 296.

(2) Jover, J.; Fey, N. Chem. - Asian J. 2014, 9, 1714.

(3) Bess, E. N.; Bischoff, A. J.; Sigman, M. S. Proc. Natl. Acad. Sci. U. S. A. 2014, 111, 14698.

(4) Harper, K. C.; Bess, E. N.; Sigman, M. S. Nat. Chem. 2012, 4, 366.

(5) Harper, K. C.; Vilardi, S. C.; Sigman, M. S. J. Am. Chem. Soc. 2013, 135, 2482.

(6) Knowles, W. S. Acc. Chem. Res. 1983, 16, 106.

(7) Tolman, C. A. Chem. Rev. 1977, 77, 313.

(8) Hansch, C.; Leo, A.; Taft, R. W. Chem. Rev. 1991, 91, 165.

(9) Charton, M.; Charton, B. J. Am. Chem. Soc. 1975, 97, 6472.

(10) Charton, M. Chem. Technol. 1975, 245.

(11) Verloop, A.; Hoogenstraaten, W.; Tipker, J. Development and Appication of New Steric Substituent parameters in Drug Design 1976, 165.

(12) Verloop, A.; Tipker, J. The STERIMOL Approach to Drug Design; Marcel Dekker: New York, 1987.

(13) Poater, A.; Cosenza, B.; Correa, A.; Giudice, S.; Ragone, F.; Scarano, V.; Cavallo, L. Eur. J. Inorg. Chem. 2009, 2009, 1759.

(14) Cavallo, L.; Correa, A.; Costabile, C.; Jacobsen, H. J. Organomet. Chem. 2005, 690, 5407.

(15) Poater, A.; Ragone, F.; Giudice, S.; Costabile, C.; Dorta, R.; Nolan, S. P.; Cavallo, L. Organometallics 2008, 27, 2679.

(16) Hillier, A. C.; Sommer, W. J.; Yong, B. S.; Petersen, J. L.; Cavallo, L.; Nolan, S. P. Organometallics 2003, 22, 4322.

(17) Lipkowitz, K. B.; D’Hue, C. A.; Sakamoto, T.; Stack, J. N. J. Am. Chem. Soc. 2002, 124, 14255.

(18) Angermund, K.; Baumann, W.; Dinjus, E.; Fornika, R.; Gorls, H.; Kessler, M.; Kruger, C.; Leitner, W.; Lutz, F. Chem. - Eur. J. 1997, 3,755 .

(19) Harper, K. C.; Sigman, M. S. J. Org. Chem. 2013, 78, 2813.

(20) Poater, A.; Ragone, F.; Mariz, R.; Dorta, R.; Cavallo, L. Chem. Eur. J. 2010, 16, 14348.

(21) Ragone, F.; Poater, A.; Cavallo, L. J. Am. Chem. Soc. 2010, 132, 4249.

(22) Wu, L. L.; Falivene, L.; Drinkel, E.; Grant, S.; Linden, A.; Cavallo, L.; Dorta, R. Angew. Chem., Int. Ed. 2012, 51, 2870.
(23) Queval, P.; Jahier, C.; Rouen, M.; Artur, I.; Legeay, J. C.; Falivene, L.; Toupet, L.; Crevisy, C.; Cavallo, L.; Basle, O.; Mauduit, M. Angew. Chem., Int. Ed. 2013, 52, 14103.

(24) Wucher, P.; Caporaso, L.; Roesle, P.; Ragone, F.; Cavallo, L.; Mecking, S.; Gottker-Schnetmann, I. Proc. Natl. Acad. Sci. U. S. A. 2011, 108, 8955.

(25) Ahmed, S. M.; Poater, A.; Childers, M. I.; Widger, P. C. B.; LaPointe, A. M.; Lobkovsky, E. B.; Coates, G. W.; Cavallo, L. J. Am. Chem. Soc. 2013, 135, 18901.

(26) Di Giovanni, C.; Poater, A.; Benet-Buchholz, J.; Cavallo, L.; Solà, M.; Llobet, A. Chem. - Eur. J. 2014, 20, 3898.

(27) Falivene, L.; Cavallo, L.; Talarico, G. ACS Catal. 2015, 5, 6815.

(28) Lattanzi, A.; De Fusco, C.; Russo, A.; Poater, A.; Cavallo, L. Chem. Commun. 2012, 48, 1650.

(29) Desimoni, G.; Faita, G.; Jørgensen, K. A. Chem. Rev. 2006, 106, 3561.

(30) Pfaltz, A.; Drury, W. J. Proc. Natl. Acad. Sci. U. S. A. 2004, 101, 5723.

(31) Seebach, D.; Beck, A. K.; Heckel, A. Angew. Chem., Int. Ed. 2001, 40, 92.

(32) Tang, W.; Zhang, X. Chem. Rev. 2003, 103, 3029.

(33) Trost, B. M.; Van Vranken, D. L. Chem. Rev. 1996, 96, 395.

(34) Yoon, T. P.; Jacobsen, E. N. Science 2003, 299, 1691.

(35) Jacobsen, E. N. In Comprehensive Organometallic Chemistry II; Wilkinson, G., Stone, F. G. A., Abel, E. W., Hegedus, L. S., Eds.; Pergamon: New York, 1995; Vol. 12, Chapter 11.1.

(36) van Leeuwen, P. W. N. M.; Kamer, P. C. J.; Reek, J. N. H.; Dierkes, P. Chem. Rev. 2000, 100, 2741.

(37) Frisch, M. J.; et al. Gaussian09; Gaussian, Inc.: Wallingford, CT, 2009.

(38) Becke, A. D. Phys. Rev. A: At., Mol., Opt. Phys. 1988, 38, 3098.

(39) Schaefer, A.; Huber, C.; Ahlrichs, R. J. Chem. Phys. 1994, 100, 5829.

(40) Leininger, T.; Nicklass, A.; Stoll, H.; Dolg, M.; Schwerdtfeger, P. J. Chem. Phys. 1996, 105, 1052.

(41) Cavallo, L.; Jacobsen, H. Eur. J. Inorg. Chem. 2003, 2003, 892.

(42) Cavallo, L.; Jacobsen, H. J. Phys. Chem. A 2003, 107, 5466.

(43) Cavallo, L.; Jacobsen, H. J. Org. Chem. 2003, 68, 6202.

(44) Jacobsen, H.; Cavallo, L. Chem. - Eur. J. 2001, 7, 800.

(45) Cavallo, L.; Jacobsen, H. Angew. Chem., Int. Ed. 2000, 39, 589.

(46) Bondi, A. J. Phys. Chem. 1964, 68, 441.

(47) Rowland, R. S.; Taylor, R. J. Phys. Chem. 1996, 100, 7384.

(48) SambVca 2.0: a web application for analyzing catalytic pockets. https://www.molnac.unisa.it/OMtools/sambvca2.0/.

(49) Brownlee, J.; He, P.; Moran, G. R.; Harrison, D. H. Biochemistry 2008, 47, 2002.

(50) Pratter, S. M.; Konstantinovics, C.; Di Giuro, C. M. L.; Leitner, E.; Kumar, D.; de Visser, S. P.; Grogan, G.; Straganz, G. D. Angew. Chem., Int. Ed. 2013, 52, 9677.

(51) Dierkes, P.; van Leeuwen, P. W. N. M. J. Chem. Soc., Dalton Trans. 1999, 1519.

(52) Deng, L.; Woo, T. K.; Cavallo, L.; Margl, P. M.; Ziegler, T. J. Am. Chem. Soc. 1997, 119, 6177.

(53) Balzani, V.; Juris, A.; Venturi, M.; Campagna, S.; Serroni, S. Chem. Rev. 1996, 96, 759.

(54) Sala, X.; Maji, S.; Bofill, R.; García-Antón, J.; Escriche, L.; Llobet, A. Acc. Chem. Res. 2014, 47, 504.

(55) Tshuva, E. Y.; Goldberg, I.; Kol, M. J. Am. Chem. Soc. 2000, 122, 10706.

(56) Resconi, L.; Cavallo, L.; Fait, A.; Piemontesi, F. Chem. Rev. 2000, 100, 1253.

(57) Corradini, P.; Guerra, G.; Cavallo, L. Acc. Chem. Res. 2004, 37, 231.

(58) Ewen, J. A. J. Mol. Catal. A: Chem. 1998, 128, 103.

(59) Cavallo, L.; Corradini, P.; Guerra, G.; Vacatello, M. Polymer 1991, 32, 1329.

(60) Cavallo, L.; Corradini, P.; Guerra, G.; Vacatello, M. Macromolecules 1991, 24, 1784. 
(61) Zuo, W.; Lough, A. J.; Li, Y. F.; Morris, R. H. Science 2013, 342, 1080.

(62) Noyori, R. Angew. Chem., Int. Ed. 2002, 41, 2008.

(63) Choroba, O. W.; Williams, D. H.; Spencer, J. B. J. Am. Chem. Soc. 2000, 122, 5389.

(64) Hubbard, B. K.; Thomas, M. G.; Walsh, C. T. Chem. Biol. 2000, 7, 931.

(65) Lagaditis, P. O.; Sues, P. E.; Sonnenberg, J. F.; Wan, K. Y.; Lough, A. J.; Morris, R. H. J. Am. Chem. Soc. 2014, 136, 1367.

(66) Prokopchuk, D. E.; Morris, R. H. Organometallics 2012, 31, 7375.

(67) Gunanathan, C.; Ben-David, Y.; Milstein, D. Science 2007, 317, 790.

(68) Zhang, J.; Leitus, G.; Ben-David, Y.; Milstein, D. J. Am. Chem. Soc. 2005, 127, 10840. 